University of South Carolina

Scholar Commons

$11-30-2020$

\title{
"When It's Time to Come Together, We Come Together": Reconceptualizing Theories of Self-efficacy for Health Information Practices Within LGBTQIA+ Communities
}

\author{
Alexander N. Vera \\ University of South Carolina - Columbia, veraan@email.sc.edu \\ Travis L. Wagner \\ University of South Carolina - Columbia, wagnertl@email.sc.edu \\ Vanessa L. Kitzie \\ University of South Carolina - Columbia, kitzie@mailbox.sc.edu
}

Follow this and additional works at: https://scholarcommons.sc.edu/libsci_studentpubs

Part of the Health Sciences and Medical Librarianship Commons, Lesbian, Gay, Bisexual, and Transgender Studies Commons, and the Psychology Commons

\footnotetext{
Publication Info

Postprint version. Published in Roles and Responsibilities of Libraries in Increasing Consumer Health Literacy and Reducing Health Disparities, Volume 47, 2020, pages 263-282.

(c) Emerald Publishing Limited, 2021
}

This Book Chapter is brought to you by the Information Science, School of at Scholar Commons. It has been accepted for inclusion in Student Publications by an authorized administrator of Scholar Commons. For more information, please contact digres@mailbox.sc.edu. 
RUNNING HEAD: "When it's time to come together, we come together"

"When it's time to come together, we come together": Reconceptualizing theories of selfefficacy for health information practices within LGBTQIA+ communities

\author{
A. Nick Vera \\ Travis L. Wagner \\ Vanessa L. Kitzie \\ University of South Carolina
}


RUNNING HEAD: "When it's time to come together, we come together"

\begin{abstract}
This chapter addresses the shortcomings of current self-efficacy models describing the health information practices of LGBTQIA+ communities. Informed by semi-structured interviews with 30 LGBTQIA+ community leaders from South Carolina, findings demonstrate how their selfefficacy operates beyond HIV/AIDS research while complicating traditional models that isolate an individual's health information practices from their abundant communal experiences. Findings also suggest that participants engage with health information and resources in ways deemed unhealthy or harmful by healthcare providers. However, such practices are nuanced, and participants carefully navigate them, balancing concerns for community safety and well-being over traditional engagements with healthcare infrastructures. These findings have implications for public and health librarianship when providing LGBTQIA+ communities with health information. Practitioners must comprehend how the collective meanings, values, and lived experiences of LGBTQIA+ communities inform how they create, seek, share, and use health information to engage in successful informational interventions for community health promotion. Otherwise, practitioners risk embracing approaches that apply decontextualized, deficit-based understandings of these health information practices, and lack community relevance.
\end{abstract}

Keywords: self-efficacy; LGBTQIA+; public libraries; health information practices 
RUNNING HEAD: "When it's time to come together, we come together"

\section{Introduction}

Self-efficacy is an integral model applied in health and library contexts to describe, predict, and influence health behavior change. This model envisions a person's potential for behavior change as shaped by beliefs in their ability, or self-efficacy, to effect this change (Bandura, 1977). Applications of this model often measure an individual's degree of self-efficacy by their use of resources created within healthcare spaces and frameworks. For example, an individual might demonstrate a high degree of self-efficacy to lose weight because they engage in exercise and diet programs recommended by their doctor. Such applications presume all behaviors prescribed by traditional healthcare spaces and frameworks to be universally positive. This presumption fails to address how an individual's misuse or rejection of resources may be potentially efficacious for alternative reasons. Lesbian, gay, bisexual, transgender, queer, intersex, and asexual (LGBTQIA+) communities have learned to navigate a healthcare and health information culture that assumes heterosexuality in alternate ways that affirm their LGBTQIA+ identities. How they create, seek, share, and use health information counters traditional framings of selfefficacy and illustrates that self-efficacy is hardly universal. Instead, it "relates to beliefs about capabilities of performing specific behaviors in particular situations" (Strecher, McEvoy Devellis, Becker, \& Rosenstock, 1986, p. 74, emphasis added).

This chapter examines how traditional self-efficacy models reify deficit frameworks that locate health challenges as indicators of individual and community failures, stigmatizing LGBTQIA+ communities, and overlooking their existing health-forward actions. Public health policy that places the responsibility of avoiding disease on individuals (Morgan \& Ziglio, 2007; Ziglio, Hagard, \& Griffiths, 2000) and library information science (LIS) research and practice that assume library users are "needy" of information and expert intervention (Frohmann, 1992; 
RUNNING HEAD: "When it's time to come together, we come together"

Julien, 1999; Olsson, 2005) illustrate these frameworks. Such uncritical frameworks assume that institutions like hospitals and libraries offer neutral, unbiased, and equitable services, and do not consider how or why individuals may avoid engaging with experts or practitioners beyond this avoidance representing a personal failing.

To counteract such limited deficit-based applications, we propose an information practices approach to reframe self-efficacy among LGBTQIA+ communities. Our ongoing research examining the health information practices of LGBTQIA+ communities in South Carolina (SC) informs this argument. Information practices represent "a set of socially and culturally established ways to identify, seek, use, and share information," unique to a given individual and their surroundings (Savolainen, 2008, p. 2). Following our re-conceptualization of self-efficacy, we address its implications for public and health librarianship. We offer practical suggestions and strategies for how practitioners can reframe deficit-based thinking concerning efficacy within LGBTQIA+ communities. These implications challenge professionals to depart from value systems that create "good" or "bad" health information practices and instead adopt values embedded within LGBTQIA+ communities.

\section{Background}

\section{Health of LGBTQIA+ Populations}

LGBTQIA+ people experience greater health disparities compared to their cisgender (i.e., individuals whose gender identity aligns with their sex assigned at birth) and heterosexual peers. Researchers have used minority stress theory as a framework to understand how these disparities manifest. Minority stress theory suggests that minority populations experience prejudice and discrimination, which in turn produces constant stress on the individual, resulting in poorer 
RUNNING HEAD: "When it's time to come together, we come together"

health overall (Katz-Wise, Mereish, \& Woulfe, 2017). Research has indicated that minority stress is associated with both physical and mental health disparities, such as the increased risk of obesity and chronic illness, higher rates of HIV infections and STIs, and enhanced mental distress (Bostwick \& Dodge, 2019; Dodge et al., 2016; Hughes, Damin, \& Heiden-Rootes, 2017; Reisner et al., 2016). Minority stress theory illustrates how health disparities are not produced by individuals being LGBTQIA+, but instead through systematic barriers that produce inequity (Katz-Wise et al., 2017). Examples of these barriers are lack of LGBTQIA+ education received by medical professionals (Lerner \& Robles, 2017), discrimination and provider insensitivity, and anti-LGBTQIA+ cultural climates (Romanelli \& Hudson, 2017). These barriers deter LGBTQIA+ individuals from seeking appropriate and timely health care because they believe that providers are unable or unwilling to care for them (Lerner \& Robles, 2017; Romanelli \& Hudson, 2017).

Like all discussions of oppression and minoritizing, it is essential to acknowledge that such systemic barriers occur intersectionally (Crenshaw, 1990). Intersectionality acknowledges that an individual does not experience any given identity in isolation, but instead lives with multiple experiences of social difference centered in identities such as race, class, age, and ability. Within the umbrella of LGBTQIA+ identities, transgender individuals delay seeking healthcare at a rate $30 \%$ higher than cisgender individuals. These delays increase for transgender women of color and lower-class transgender women (Lerner \& Robles, 2017). This example illustrates the particular intensity of barriers to access for LGBTQIA+ persons navigating health care. These barriers produce new stressors and challenges compounded along the lines of racial and class-based inequity, among other salient intersectional identities. To promote their health, 
RUNNING HEAD: "When it's time to come together, we come together"

LGBTQIA+ individuals must face these obstacles despite potential risks or actual harm, which necessitates strong belief in their abilities (i.e., self-efficacy) to overcome these challenges.

\section{Self-Efficacy}

Self-efficacy is a concept within social cognitive theory that describes how people's beliefs in their abilities shape their motivations for behavior change (Bandura, 1989). An individual develops self-efficacy from their environment and personal knowledge, which includes social interactions, personal experiences, and external influencers. Traditional applications of selfefficacy seek to understand and predict whether people might exhibit a specific behavior within a particular socio-cultural context (Bandura, 1989). The ultimate goal of these applications is to identify, shape, and ultimately change people's behaviors to elicit a "positive" outcome as defined by researchers, such as eating more vegetables or increasing exercise (Schwarzer \& Warner, 2013). Self-efficacy literature suggests that those who possess higher levels of selfefficacy are more likely to engage in positive behavior changes. Those with lower self-efficacy may self-sabotage their success, resulting in negative behavior change (Bandura, 1977).

Typical health contexts for self-efficacy focus on smoking (DiClemente, 1981), exercise (Marcus, Eaton, Rossi, \& Harlow, 1994), and diet (Chair, Wong, Tang, Wang, \& Cheng, 2015). Self-efficacy has also been adopted by LIS research to determine literacy levels. Existing work explores whether individuals possess "adequate" efficacy regarding information literacy (again, defined by researchers), such as their ability to locate, appraise, and effectively use the information required to make necessary decisions, and frames "fixes" to this lack of efficacy through the utilization of library services (Usluel, 2007). Existing systematic reviews on selfefficacy among LGBTQIA+ populations focus almost exclusively on HIV/AIDS prevention and condom use, centering on men who have sex with men (Weinhardt, Forsyth, Carey, Jaworski, \& 
RUNNING HEAD: "When it's time to come together, we come together"

Durant, 1998). More recent research has expanded this exploration to issues such as smoking cessation (Berger \& Mooney-Somers, 2017), correlations between substance abuse and sexual risk (Knight et al., 2019), and homelessness among transgender populations (McCann \& Brown, 2018).

Self-efficacy research often develops universal scales for use across diverse populations to understand the relationship between self-efficacy and behavior change. The universality of these scales is problematic as certain groups become targets for increased interventions due to their high self-efficacy levels. In contrast, others can be deemed unfit for intervention due to their low self-efficacy levels (Schwarzer \& Renner, 2009). Further, the scaling of efficacy overlooks cases in which an individual's low self-efficacy in one context might indicate high self-efficacy elsewhere. For example, an individual may exhibit high self-efficacy to quit smoking by engaging in routines that minimize opportunities to smoke during the workday yet have low self-efficacy in social environments such as a smoking-heavy bar. Scales here would mark the individual's efficacy as insufficient to them following through with complete smoking cessation. Further, self-efficacy is not universal and can vary across specific groups. For instance, a study of homelessness and sex work among incarcerated women found that without a stable means to meet their survival needs, many women engaged in risky behaviors such as exchanging sex for drugs or housing (Kim, Johnson, Goswami, \& Puisis, 2011). While researchers and society perceive these actions to be harmful, we argue that these women possess high self-efficacy because their beliefs in their abilities to meet their financial, housing, and healthcare needs are strong. These strong beliefs allow them to acquire the necessary resources needed to survive despite extreme hindrances. 
RUNNING HEAD: "When it's time to come together, we come together"

Current self-efficacy models also fail to address the harsh realities faced by LGBTQIA+ populations when accessing health care and instead adopt heteronormative frameworks that assume or equate LGBTQIA+ health needs with those of similar-aged heterosexual or cisgender peers (Colpitts \& Gahagan, 2016). These models also ignore deliberate agentic choices made by LGBTQIA+ individuals to not engage with traditional health care spaces and services, marking these choices as indicative of low self-efficacy concerning behavior change. Our findings complicate and extend this work by demonstrating how LGBTQIA+ persons purposefully engage with community-centric health information spaces outside of traditional venues, which challenges conventional applications of self-efficacy.

This discussion highlights the importance of contextualizing self-efficacy for a particular individual or community while also considering that an individual exhibiting self-efficacy in one arena of life is not the same as exhibiting it in another. Our work attends to these ideas by contextualizing self-efficacy within a community context. Although theorists traditionally consider self-efficacy to be separate from a community or collective efficacy, we purposefully extend this definition to incorporate community efficacy. We envision both forms of efficacy as co-constitutive. Current studies define community efficacy as a community-based judgment (Sampson, Morenoff, \& Gannon-Rowley., 2002), building upon Bandura's (1995) definition of “one's capabilities to organize and execute courses of action required to manage prospective situations" (p. 2). Our choice to replace "collective" with "community" is deliberate. As we will later show in our findings, our participants value "community" as both a term and idea. Our research also unsettles the positive and negative use of health information, through considering the efficacy of health information practices within LBTQIA+ individuals (self) and among others who share their identity and space (community). 
RUNNING HEAD: "When it's time to come together, we come together"

\section{Methodology}

This research is part of a more extensive investigation (University of South Carolina IRB approval number Pro0008587) examining the health information practices of LGBTQIA+ communities. Therefore, we only discuss our methods and present findings relevant to the focus of this chapter - the relationship between self-efficacy and the information practices of SC LGBTQIA+ communities. We conducted semi-structured interviews with 30 SC LGBTQIA+ community leaders. We chose leaders because they possess a bird's eye view of their communities. Examples of leadership roles held by participants include grassroots activists, LGBTQIA+ center board members, and cabinet members of high school Gay-Straight Alliances. We defined community as possessing three criteria: 1) members do the majority of their work in SC, 2) this work is social and involves group-oriented engagements, and 3) members collectively possess LGBTQIA+ identities (Hillary, 1995).

Informed by these criteria, we developed a spreadsheet of over 100 SC LGBTQIA+ groups and affinity organizations. We contacted these organizations via email, asking them to self-nominate leaders for participation. We also asked interviewees to recommend additional participants. Finally, we used theoretical sampling to identify participants from informal communities, such as social media-based LGBTQIA+ groups, which we may not have identified in our initial purposive sampling. Identifying these groups was vital because they may have experiences overlooked within popular LGBTQIA+ narratives.

Interview topics included participants' involvement with their communities, their personal and community identities, and how they and their communities addressed their health questions and concerns. Interviews were followed by an information world mapping exercise 
RUNNING HEAD: "When it's time to come together, we come together"

that asked participants to draw the people, places, and things that helped or did not help them address their health questions and concerns. This visual elicitation method triangulated interview data, providing additional insights into topics covered by the semi-structured protocol (Greyson, 2013). See Appendix A for links to supplemental materials, including the study protocol and information worlds mapping exercise.

Data sources included interview transcripts, information worlds maps, observational notes, and reflexivity journals maintained by the research team. This chapter will focus exclusively on the analysis of interview transcripts supplemented by observational notes. Data analysis used a deductive coding process to develop a provisional list of codes established in the self-efficacy literature and informed by the above literature review. These codes are selfefficacy, response efficacy, community efficacy, and community response efficacy (See table 1 below for code definitions).

\begin{tabular}{|l|l|}
\hline Table 1: Efficacy Codebook Definitions \\
\hline Code & Definition \\
\hline Community Efficacy & $\begin{array}{l}\text { A community-based judgment of how well they can } \\
\text { execute courses of action required to deal with } \\
\text { prospective situations. }\end{array}$ \\
\hline Community Response Efficacy & $\begin{array}{l}\text { A community's belief that the identifiable action } \\
\text { step will achieve the target goal or objective. }\end{array}$ \\
\hline Self-Response Efficacy & $\begin{array}{l}\text { An individual's belief that the identifiable action } \\
\text { step will achieve the target goal or objective. }\end{array}$ \\
\hline Self-Efficacy & $\begin{array}{l}\text { An individual's judgment of how well they can } \\
\text { execute courses of action required to deal with } \\
\text { prospective situations. }\end{array}$ \\
\hline
\end{tabular}

We applied these four codes to our data sources, using sentences as our unit of observation. Our research team worked in unison to code three transcripts, actively addressing 
RUNNING HEAD: "When it's time to come together, we come together"

questions and coding discrepancies. Following this process, we divided the remainder of the transcripts and applied these deductive codes. Then we engaged in member-checking, sending participants their transcripts, as well as our field notes and a draft write-up of our findings for their review. We asked participants to remove any remaining identifying information and let us know how well they felt our data and preliminary reporting reflected their lived experiences (Creswell \& Poth, 2016).

\section{Participant Demographics}

We asked participants to select their age from a list of ranges; the majority indicated they were either young adults (ages 18-25: $n=11 ; 36.7 \%$ ) or middle-aged adults (ages 35-54: $n=7 ; 23.3 \%$ ). We also interviewed 4 participants in the 13 to 17 -year-old range, as we felt these leaders provided crucial insight into the experiences of young LGBTQIA+ communities. Participants also selected from a series of racial and ethnic identities with the ability to add identities not listed. Broadly, the racial and ethnic background of participants represented state demographics for LGBTQIA+ populations and racial minorities in SC (Williams Institute, 2018). Most participants reported having some level of higher education. Distribution of participants varied across the state, with higher representation from the Upstate and Midlands regions.

Participants identified across the spectrum of LGBTQIA+ identities, with a particular prevalence of lesbian, gay, queer, transgender, genderqueer, and bisexual identities. Given the complexity around the labeling of LGBTQIA+ identities, we asked participants to self-label as opposed to choosing from a series of labels. This data collection strategy afforded our participants the ability to express their gender identities and sexual orientations in any way that they saw fit, particularly since there exist multiple ways people can identify as transgender and 
RUNNING HEAD: "When it's time to come together, we come together"

bisexual. Even when participants evoked similar labels, we believe it unethical to group said identifications as the same. For further information on methods used to cull demographic information, refer to Kitzie, Wagner, and Vera (2020).

\section{Findings}

The four deductive codes we applied to participant narratives illustrate three significant findings from our research: 1) information and self-efficacy inform each other and shape information practices at individual and community levels, 2) LGBTQIA+ individuals communally deploy self-efficacy, and 3) self-efficacy is not a universal concept nor does a universal standard exist for measuring its value. We will illustrate these findings using participant narratives that exemplify our four deductive codes. We refer to participants in this section using their self-selected pseudonyms and provided pronouns. When a participant uses multiple pronouns, such as he/him/his and she/her/hers, we switch between them.

\section{Finding 1: Information and Self-Efficacy as Co-Constitutive}

Our first narrative from participant Tony illustrates finding one (information and self-efficacy are co-constitutive of one another) using three codes: (1) Community Response Efficacy; (2) Community Efficacy; and (3) Self-Response. Tony is a black transgender man who works as a grassroots community leader, advocating and aiding transgender individuals in the process of socially and medically transitioning. During his interview, Tony recounted an example of his community coming together to prevent the potential harm caused by a pending bathroom bill in SC. This bill would force individuals to use the bathroom indicated by the gender marker on government-assigned documents. As Tony observed, such bills would prove detrimental to the safety and rights of SC transgender individuals. 
RUNNING HEAD: "When it's time to come together, we come together"

Tony recalled cautiously attending a hearing at the SC statehouse to vote on the bill. His caution motivated by a concern that he and members of his community might be facing a losing battle, especially given a similar bill's passing months earlier in North Carolina (North Carolina Legislative Assembly House Bill 2, 2016). Tony assumed that, like himself, his community had ceased to "pay attention to politics" and doubted that members would attend the hearing. However, to Tony's surprise, many individuals from his community planned to attend and insisted that he join them. He noted he had "never been to something like that," and the community turnout against the bill was unprecedented. These observations made Tony realize that, for his community, "when it's time to come together, we come together." Inspired by the courage and tenacity of his community, Tony then stated, "I don't know what we're going to do [next], but I want to do it together." One contribution Tony made to subsequent events concerning the bathroom bill was printing fliers to protest the bill and handing them out to supporters.

Two fundamental forms of efficacy are at work in this narrative. First, Tony expressed surprise at his community's response efficacy. Specifically, Tony's decision to join his community in protesting the bathroom bill counteracted his assumptions about the power of homophobia and transphobia to deter such action. Tony's past experiences with transphobic legislation led him to believe his advocacy to be futile. However, when his community insisted on showing up at the statehouse, he felt reinvigorated to advocate against such bills occurring in the future. Tony's experience with his community's response efficacy motivated his self-response efficacy, in which he identified ways to help, such as printing fliers. The positive collective response by Tony's community helped enhance his activism by rekindling his passion for it. Tony imagined how his visible activism might become equally infectious in his community, 
RUNNING HEAD: "When it's time to come together, we come together"

resulting in a chain of efficacious actions toward increased visibility and community efficacy among SC transgender communities.

Other instances supporting our finding that information and self-efficacy shape individual and community practices co-constitutively occurred in participant interviews offering insight into the intersectionality of LGBTQIA+ identities. For instance, Vada, who leads a youth social group at her local LGBTQIA+ community center, started the group based on her personal experiences of isolation from people like her. Vada presumed that others might feel the same:

I realized I didn't really fit in with a lot of the kids... I wasn't fitting in with people... And I was becoming depressed about the fact that I really had nowhere else to go because I've been online schooled.... So, I really had nothing, because the center had nothing... So I decided that I might as well take the effort to do it myself...And so that's how it started.

Though Vada's and Tony's experiences are drastically different in context, they share similar anti-LGBTQIA+ barriers. For Tony, it was seeing his community converging against continued regulatory legislation that sparked his refusal to let anti-transgender sentiment remain normal. Tony drew on his self-response efficacy to build tangible practices to improve the community efficacy of those around him. Similarly, the same can be argued for Vada, as she responded to the lack of available resources and imagined that others experienced similar challenges posed by this lack of resources. Vada's self-response efficacy motivated the center's community response efficacy in addressing the issue directly by creating an LGBTQIA+ youth group. By creating this group, both Vada and the center took actionable steps to create visibility and community efficacy for LGBTQIA+ youth.

\section{Finding 2: LGBTQIA+ Individuals Deploy Self-Efficacy Communally}


RUNNING HEAD: "When it's time to come together, we come together"

Our next set of narratives illustrate another finding, which is that participant beliefs, motivations, and actions that may initially appear to be self-efficacious operate as community efficacy. Ben, a gay high school student, illustrates this finding when he recalled meeting with other LGBTQIA+ classmates after school, "near our garden [against] the brick wall." One subject of conversation among them were their various health-related information needs, such as the need for more information about PrEP (i.e., a preventative HIV medicine). According to Ben, "A lot of people have PrEP, but we don't know how old you have to be, to be on PrEP [...] it literally doesn't say on the website." After discussing their health-related needs, the community would gather "all [their] questions like, 'Who's going to the doctor's appointment?' The person with the next doctor's appointment would take the collected questions and ask them at their visit, returning to the community with the answers.

While the practice of visiting the doctor and asking a health-related question appeared to exhibit self-efficacy, in context, it represented community efficacy. Several barriers and challenges rendered it difficult for Ben and her community to independently visit doctors and ask health-related questions. These barriers included not being out to one's family, lacking insurance coverage, and not having an LGBTQIA+ friendly primary care physician. Further, these multiple underlying barriers had the potential to produce stressors among Ben's community that could have consequences for members' physical and mental health. However, Ben's community constructed solutions to satisfy their health information needs, even if circuitous and unconventional. These practices attended not to medical authority, but instead to community comfort and safety. While it is easy to perceive these practices as unsafe and ineffective within public health discourses, they reflect the hyper-specialized needs of Ben and her community and exhibit high community efficacy. 
RUNNING HEAD: "When it's time to come together, we come together"

Another example of community efficacy discussed by Ben is how her community forms private groups on social media, specifically Snapchat, to share relevant health research and information: "We read a lot of articles together. We share articles. We can read articles about tips on being sexually healthy and all that other stuff... If we find something interesting, we refer it to someone else." This example illustrates community efficacy through alternative uses of social media by community members to combat existing homophobia and transphobia. In particular, Ben's group displays community efficacy through their circulation of resources, which occurs despite systemic barriers produced by educators, family, health care providers, and physicians. This collective practice serves as a tactical way for Ben and her community to avoid surveillance by using Snapchat features like automatic message deletion.

Jordan, a young, gay black man attending a Historically Black College and University and leading a group for other young, black gay men, shared the challenges that he and his community face due to their university's refusal to recognize their presence on campus. Jordan takes it upon himself to defend his community from these challenges through actions like protesting, promoting pro-LGBTQIA+ events online, and by voicing his community's concerns to powerful university administrators:

[I] use any platform that I have to allow everyone's voice to be heard, especially those that are not heard ... when my fellow students voice their opinions or their concerns, it's not heard... I take on the role of doing that because people listen to me and I can get into those safe spaces by being the voice or the mouthpiece for those people.

Jordan embodies both self and community efficacies to help him and his community thrive. He exhibits self-efficacy by acknowledging his straight-passing privilege, despite being a gay man, and using it to voice his community's concerns. These actions represent Jordan's judgments on 
RUNNING HEAD: "When it's time to come together, we come together"

the extent to which he can temporarily bypass spaces that exclude him in order to fulfill his role as a community leader, thereby also exhibiting community efficacy.

Jordan's narrative reveals not only the multiple efficacies used to navigate challenges and barriers at his university but also the multiplicity of barriers he and his community face. For Jordan, these underlying barriers shape his community's perception of university spaces as unwelcoming, such as campus spaces where students can voice their concerns directly to higher administration. Before Jordan's work, his community avoided entering such spaces in fear of potential repercussions brought on by the university. Similar to Ben's and her community's efficacy to develop health information practices outside of institutionalized surveillance, Jordan undertakes similar actions. He uses his visibility to force his institution to take seriously the needs and values unique to his community, which had previously gone unacknowledged.

\section{Finding 3: Self-Efficacy is not a Universal Concept}

Our next participant narrative represents our third findings, which posits that self-efficacy is not a universal concept, nor does a universal standard exist for measuring its value. To illustrate this finding, Pat, a transgender woman of color, discussed her and her community's experiences accessing reliable health care. Similar to Ben's community, the practices that Pat and her community engage in appear discursively negative at face value. One notable example Pat provided was "bugchasing," or the act of deliberately contracting HIV/AIDS to access health care. While bugchasing represents an individualized practice, Pat's community exhibits and shares common knowledge of what it is and how they can use it to access care. In particular, Pat speaks about obtaining a status of "positive" (i.e., testing positive for HIV/AIDs) and the points of access it provides: 
RUNNING HEAD: "When it's time to come together, we come together"

So a lot of people that I share a community with, a lot of us don't have health insurance or don't have the capacity to afford health insurance, and so there are several different ways that we access health care... Some of us are able to access healthcare if we're positive, so if we're positive, we can go to places like the immunology center, and we're able to get on like treatment regimens, and other federally-funded programs are able to get us dental care, and healthcare... There are some people who are referred to as bugchasers, who try to become positive in order to access those things as well...

Pat's account illustrates why community members engage in bugchasing - to access healthcare and other resources despite structural barriers, such as a lack of health insurance or the inability to afford insurance. Pat further located community motivations for bugchasing as being tied to members' shared identities as Black LGBTQIA+ persons who experience anti-LGBTQIA+ sentiment and racism daily. She elaborated on these experiences as "structural problems [that] either encourage or promote unhealthy practices, subjectively defined by me... that inhibit our capacity to access healthcare, even in the situations where we would prefer to take those routes." Pat's community may prefer using mainstream methods to access health care; however, because of structural barriers, they are often left with no other "routes," rendering bugchasing a viable means to access care.

Pat's narrative contrasts with traditional frameworks of self-efficacy by illuminating the exacerbating role negative experiences play concerning accessing care in her community. In the context of Pat's experiences, we argue that structural barriers that prevent access to mainstream methods of care shape Pat and her community's self and community response efficacy. As a result, bugchasing becomes an efficacious practice that produces the intended goal of accessing health care, despite the risk of contracting HIV/AIDs. 
RUNNING HEAD: "When it's time to come together, we come together"

Other interview participants also demonstrate alternative routes used by LGBTQIA+ individuals and community members to obtain care. For instance, Tony discusses challenges experienced by those in his community who are not covered by insurance but are in the process of transitioning from their sex assigned at birth. For Tony's community, part of this transitioning includes the use of hormone treatments. As he explains, insurance, visiting a doctor, and being able to obtain hormone prescriptions are "big issues" for his community. Tony discusses his own experiences affording hormones without insurance, noting his “hormones went from a month's supply for five bucks to a week's supply for $\$ 90, "$ creating serious challenges for Tony and those like him who are transitioning without any reliable health insurance. Tony noted that these challenges influence the decisions of some community members to engage in extralegal methods to obtain hormones, looking to him as a source for purchasing or trading hormone injections without a prescription.

Tony's account illuminates the lengths community members will go to obtain hormones and the degree to which self and self-response efficacy are needed to engage in this behavior. Moreover, these instances illustrate the function of barriers operating as facilitators rather than deterrents to the community's self and community-response efficacy, thus enabling their behaviors. Attempts to obtain hormones outside of legal, medical settings also displays self and community efficacy among Tony's community members who are seeking affirmation of their gender identity despite potentially detrimental health and legal consequences. Tony's narrative supports our third key finding that self-efficacy is not and cannot be a universal concept. Some members of Tony's community who are transitioning consider their increase in self-worth and emotional well-being by taking hormones to outweigh the risks of potentially being charged with a crime and to their physical health. 
RUNNING HEAD: "When it's time to come together, we come together"

\section{Discussion and Implications}

\section{Queering Self-Efficacy Frameworks for LGBTQIA+ Communities}

An emergent theme from our research illuminates gray areas between the binary of success and failure established by self-efficacy scales. For example, Wagnild and Young's (1993) resilience scale, "identif[ies] the degree of individual resilience," needed to implement "a positive personality characteristic that enhances individual adaptation," which then becomes a signal of an individual's "ability to cope effectively when faced with adversity" (Wagnild, 2009, pp. 105167). This scale frames self-efficacy in a way that binarizes behavior into positive and negative values. These values correlate an individual's health outcomes with their adherence to predefined health behaviors. Within these frameworks, individuals possess low or high levels of self-efficacy based on whether or not they engage in practices that lower their probability of experiencing adverse health outcomes.

In contrast to these binary measures and universal assumptions of risk and health, we find that many LGBTQIA+ leaders and communities deliberately work outside of traditional information flows and complicate fixed ideas of riskiness and healthiness. The challenge of binarized logics is evident when communities oppose the presumed knowledge of health professionals and other authorities, such as educators and information professionals, in favor of knowledge produced by, and centering on, their communities. In doing so, they do not negate the value of authoritative health information, but rather aim to protect the well-being of their LGBTQIA+ peers. The reasons for engaging in less traditional approaches to access care include safety, comfort, and a desire to avoid heteronormative (i.e., a presumption by society that all individuals are heterosexual and that the world as such is designed for and caters to the needs of 
RUNNING HEAD: "When it's time to come together, we come together"

heterosexuality) and cisnormative (i.e., assuming that a person's gender matches their sex assigned at birth) cultural discourses.

To say that participants in this study possess low self-efficacy or lack the desire to succeed ignores the contextual reasons why LGBTQIA+ communities do not "correctly" engage with health information. Traditional self-efficacy frameworks incorrectly label "failure" as negative. Instead, we argue that LGBTQIA+ communities' failure to engage in health information practices marked as discursively positive constitutes an affirming, agentic, and generative set of actions. We echo Jack Halberstam's (2011) argument that failure is an inherently queer practice as it "refus[es] to acquiesce to dominant logics of power and discipline and as a form of critique" (p. 88).

Our use of "queer" works to unsettle notions of fixed binaries of any sort, regardless of their direct ties to LGBTQIA+ identities. Famous queering work often aims to unsettle ideas of low/high culture, good/bad art, and productive/unproductive work. Our participants' presumed failures work to successfully "exploit the unpredictability of ideology and its indeterminate qualities" (Halberstam, 2011, p. 88). It is not LGBTQIA+ communities who are failing to use health information, but healthcare systems that are failing to address the specific needs of LGBTQIA+ persons. LGBTQIA+ communities respond to this failure by queering health information practices to succeed despite structural barriers. An example from our findings is that the "unpredictability" of whether or not a given healthcare provider would be pro-LGBTQIA+ led Ben and her community to "exploit" the resources of one healthcare provider out of concern for community safety. As this and our earlier examples show, LGBTQIA+ communities create alternative practices that exploit the logics of medical institutions to access information and resources otherwise made inaccessible to them. In these examples, what could be seen as a 
RUNNING HEAD: "When it's time to come together, we come together"

failure within traditional concepts of self-efficacy works to de-binarize both the success and failure of actions, while blurring universalized divisions between individual and community contexts. What works for one community is not going to work for another.

Much as there is not an easily divisible line between successful and failed use of health information within LGBTQIA+ communities, so too there exists a blurring between engagement with health information by an individual and their larger community. For LGBTQIA+ persons, community efficacy and self-efficacy exist interchangeably. Once again, Ben and his community's sharing of health information with one another based on one person's doctor visit displays purposeful addressing of community health concerns without making particular individuals visible. Such deliberate navigation of healthcare spaces suggests high efficacy concerning community safety. This safety comes through enacting what health professionals might perceive to be low self-efficacy on the part of individuals within the community who "fail" to go to the doctor. While traditional frameworks of self-efficacy might label a person as having low self-efficacy, the infusion of community efficacy into the discussion tells a different story.

\section{Health Librarianship's Role in Advocating for LGBTQIA+ Health Information Practices}

Findings from our research provide guidelines for public libraries to facilitate the health information practices of LGBTQIA+ communities. Much like all shifts in librarianship, this work warrants structural alterations. First, findings from our research suggest that librarianship must reconsider its commitment to neutrality. In line with Pagowsky and Wallace (2015), we argue that, given the minority status of LGBTQIA+ individuals, practices within librarianship that are not critical (i.e., neutral) could prove "ineffective" and "even hostile" by reinforcing heteronormative and cisnormative structures (p. 199). One potentially hostile example might 
RUNNING HEAD: "When it's time to come together, we come together"

include father-daughter storytimes that presume fathers to be cisgender men, or question the presence of two fathers taking their daughter to the storytime. In turn, we argue that librarianship must shift from an objective, neutral philosophical framework, and instead, practitioners must situate their work within a community- and person-oriented praxis. Librarians, for example, should be aware that when it comes to medical research on or about transgender individuals, much of the language and findings evidence practitioner transphobia as opposed to ignorance exhibited by transgender people (Stroumsa, Shires, Richardson, Jaffee, \& Woodford, 2019). Our proposed praxis would recognize such research as detrimental, and the librarian would avoid providing it to patrons, instead seeking out alternative, inclusive resources.

Both patrons and librarians come into library spaces of all shapes and forms with their own material experiences, which influence how they see and are seen in relation to normative discourses. Take Ben and her community as an example. For him, the entirety of high school produces experiences of homophobia and transphobia. The resulting and justifiable presumption for Ben is to assume that her library also is an anti-LGBTQIA+ space. Therefore, practitioners must possess some knowledge of LGBTQIA+ identities, a more nuanced understanding of the historically complex relationships between LGBTQIA+ persons and health care professionals, and a willingness to work without judgment with such populations (Drabinski, 2018). Public librarianship must, in turn, understand that LGBTQIA+ patrons see valuable information within health and library contexts as potentially detrimental to their health and well-being.

Providing space for LGBTQIA+ persons to generate and share information is one way in which a more person-oriented approach to praxis, informed by individual needs and not by established institutional ideals, can restructure information access and community efficacy between information professionals and LGBTQIA+ populations. For example, a library could 
RUNNING HEAD: "When it's time to come together, we come together"

create an active and updated list produced by LGBTQIA+ community members that identifies local LGBTQIA+ resource's such as; businesses, clinics, events, or activities can be promoted through public libraries. Meaningful approaches such as this increase visibility for LGTBQIA+ communities and libraries, while asserting libraries as being a safe and inclusive space in which communities can seek and share valuable information produced by their community. This approach cannot stop at providing cursory services that support LGBTQIA+ persons in minimal ways, but instead requires a complete overhaul of how LGBTQIA+ exclusion exists within librarianship in ways similar to its peer institution of medicine (Wagner \& Crowley, 2020). Inclusion of services for LGBTQIA+ persons cannot merely be a singular corrective measure, such as book displays or gender-neutral bathrooms. It must entail systematic investigations into the types of exclusions and barriers that occur within such spaces.

Alterations to library-based curriculum provide a crucial avenue through which to shift the perceptions of librarianship from a neutral practice to one with an investment in personoriented, social justice-influenced frameworks. We agree that "information professionals are involved in every level of information provision and technology design," which allows for their "impact" to have community-wide influence (Cooke, Sweeney, \& Noble, 2016, pp. 120-121). Such a shift to a person-oriented, social justice curriculum affords students and future information professionals "a more holistic and inclusive perspective on the relationships between people, information, and technology" (p. 121). This curriculum privileges community needs and practices over "best practices" that center health and information professionals as the ultimate providers and arbiters of information. Through this curriculum, practitioners could help expand the field's understanding of how LGBTQIA+ communities seek, share, and use health information, both within and outside of traditional channels. They also could assist healthcare 
RUNNING HEAD: "When it's time to come together, we come together"

providers by offering space, information, and resources to leverage the robust, purposeful, and highly efficacious information practices already occurring within LGBTQIA+ communities.

As Wagner and Keeling (2019) suggest, adopting such a curricular shift compliments service-learning approaches. These approaches function as "an ideal way to get [students] to engage in experiences beyond the classroom" while "illustrat[ing] to students the complexities that arise when working within communities of which they are not a member" (pp. 355-356). Through these approaches, library students learn that service provision is hardly an isolated, onesize-fits-all practice. As our findings indicate, LGBTQIA+ communities engage self and community efficacy co-constitutively. In response, practitioners can work towards acknowledging this multiplicity by providing information not only for individualized needs but also for community ones, as informed by and perhaps even involving participation of LGBTQIA+ communities. In an alternative LIS curriculum, we would see the role of librarianship as not providing information that is right for a universal patron, but rather recognizing that what is right for one person is rarely right for another.

\section{Limitations}

As mentioned in our Methodology section, we interviewed 30 LGBTQIA+ community leaders from a variety of age groups, racial and ethnic backgrounds, educational levels, regions, and identities within the larger LGBTQIA+ umbrella. Absent from this group were leaders from "hidden" communities. These groups may experience barriers that are significantly unlike those of our participants, which may produce unique efficacious health and information practices not found among other participants. For example, our sample did not include LGBTQIA+ Latinx leaders. Latinx LGBTQIA+ groups are challenging to identify in SC, as the state's political stance and practices towards immigrants from countries within the Latinx diaspora remain 
RUNNING HEAD: "When it's time to come together, we come together"

wholly discriminatory, thus resulting in these communities remaining purposefully "hidden."

Future research aims to explore and sample LGBTQIA+ groups from other populations to extend our understanding of the forms efficacy takes within these populations.

Other limitations include the background and historical use of self-efficacy as being mostly understood from a deficit perspective, making it challenging to discuss self-efficacy without recognizing its current and past uses, and perpetuating the same damaging and stigmatizing ideologies upon LGBTQIA+ populations. Finally, our study centers around our participants' understandings and inferred meanings of the term "health." As evidenced through our data analysis process, this term is used very broadly among participants, making it difficult to distinguish its intended meaning from unintentional or alternative meanings (e.g., mental vs. physical health). In turn, we relied on field notes taken from each interview to better situate participants' use of the term "health" within both community and individual contexts and concerning any barriers described by participants. Another future research direction would be to design a protocol that identifies and clearly distinguishes between participants' meanings and understandings of health.

\section{Conclusion}

Self-efficacy frameworks have historically shaped how health and information professionals understand behavior changes toward more positive and healthier lifestyles by focusing on health, well-being, and preventative care. While engaging in these practices can be valuable for some, how professionals deploy them proves particularly damning for LGBTQIA+ persons by marking them as failed bodies needing correction. What such frameworks fail to understand are the nuanced, contextual experiences informing LGBTQIA+ health practices, both within and outside of the medical establishment. Drawing on findings from our ongoing research focused on the 
RUNNING HEAD: "When it's time to come together, we come together"

health information practices of LGBTQIA+ individuals, we suggest that not only do these communities possess high levels of self-efficacy, but also that their health information practices are deliberate and agentic.

These findings denote the importance of re-framing self-efficacy as a conceptual lens to study LGBTQIA+ health and health information practices. This re-framing must move away from binarized notions of success and failure as related to health behaviors, while simultaneously acknowledging that for many LGBTQIA+ individuals, the role of self is equal to, if not surpassed by, the community's role. Understanding community needs further expands efficacy beyond measuring an individual's isolated experience, instead envisioning health information practices as shaped by external obstacles such as stigma and discrimination.

Since public librarians remain fundamental to the production and distribution of health information, we see them as integral to shifting how health professionals and others think about working with LGBTQIA+ communities. Our findings confirm that these communities engage in extensive and positive health information seeking and sharing outside of the medical establishment. We offer a point of entry for information professionals to help amplify their voices. Further, we believe that models of success as dictated by medical professionals prove illequipped for application to LGBTQIA+ communities. Going forward, professionals must understand that there already exist remarkable health-related achievements made by LGBTQIA+ communities that grew, and continue to grow, out of the failures of healthcare professionals. In making this recognition, both LIS praxis and healthcare provision can begin to appreciate the tenacity of LGBTQIA+ communities in visibly seeking their version of being healthy in the face of continued challenges. 
RUNNING HEAD: "When it's time to come together, we come together"

\section{References}

Bandura, A. (1977). Self-efficacy: toward a unifying theory of behavioral change. Psychological Review, 84(2), 191-215.

Bandura, A. (1989). Human agency in social cognitive theory. American Psychologist, 44(9), 1175.

Bandura, A. (1995). Self-Efficacy in Changing Societies. Cambridge, UK: Cambridge University Press.

Berger, I., \& Mooney-Somers, J. (2017). Smoking cessation programs for lesbian, gay, bisexual, transgender, and intersex people: A content-based systematic review. Nicotine \& Tobacco Research, 19(12), 1408-1417.

Bostwick, W. B., \& Dodge, B. (2019). Introduction to the special section on bisexual health: Can you see us now? Archives of Sexual Behavior, 48(1), 79-87.

Chair, S. Y., Wong, K. B., Tang, J. Y. M., Wang, Q., \& Cheng, H. Y. (2015). Social support as a predictor of diet and exercise self-efficacy in patients with coronary artery disease. Contemporary Nurse, 51(2-3), 188-199.

Colpitts, E., \& Gahagan, J. (2016). The utility of resilience as a conceptual framework for understanding and measuring LGBTQ health. International Journal for Equity in Health, $15(1), 1-8$.

Cooke, N. A., Sweeney, M. E., \& Noble, S. U. (2016). Social justice as topic and tool: An attempt to transform an LIS curriculum and culture. The Library Quarterly, 86(1), 107124.

Crenshaw, K. (1990). Mapping the margins: Intersectionality, identity politics, and violence against women of color. Stanford Law Review, 43, 1241-1299. 
RUNNING HEAD: "When it's time to come together, we come together"

Creswell, J. W., \& Poth, C. N. (2016). Qualitative inquiry and research design: Choosing among five traditions. Thousand Oaks, CA: SAGE.

DiClemente, C. C. (1981). Self-efficacy and smoking cessation maintenance: A preliminary report. Cognitive Therapy and Research, 5(2), 175-187.

Dodge, B., Herbenick, D., Friedman, M. R., Schick, V., Fu, T. C. J., Bostwick, W., . . Sandfort, T. G. (2016). Attitudes toward bisexual men and women among a nationally representative probability sample of adults in the United States. PLoS One, 11(10), paper e0164430.

Drabinski, E. (2018, February 11). Are libraries neutral? [Conference Session]. American Library Association Midwinter Conference, Denver, CO, United States. Retrieved from http://www.emilydrabinski.com/are-libraries-neutral/

Frohmann, B. (1992). The power of images: A discourse analysis of the cognitive viewpoint. Journal of Documentation, 48(4), 365-386.

Greyson, D. (2013). Information world mapping: A participatory, visual, elicitation method for information practice interviews. Proceedings of the American Society for Information Science and Technology, 50(2), 1-4.

Halberstam, J. (2011). The queer art of failure. Durham, NC: Duke University Press.

Hillary, G. A. (1995). Definitions of community: Areas of agreement. Rural Sociology, 20, 111123.

Hughes, R. L., Damin, C., \& Heiden-Rootes, K. (2017). Where's the LGBT in integrated care research? A systematic review. Families, Systems, \& Health, 35(3), 308-319. 
RUNNING HEAD: "When it's time to come together, we come together"

Julien, H. (1999). Where to from here? Results of an empirical study and user-centered implications for information design. In T.D. Wilson and D.K. Allen (Eds.) Exploring the Contexts of Information Behaviour, (pp. 586-596) London: Taylor Graham.

Katz-Wise, S. L., Mereish, E. H., \& Woulfe, J. (2017). Associations of bisexual-specific minority stress and health among cisgender and transgender adults with bisexual orientation. The Journal of Sex Research, 54(7), 899-910.

Kim, S., Johnson, T. P., Goswami, S., \& Puisis, M. (2011). Risk factors for homelessness and sex trade among incarcerated women: A structural equation model. Journal of International Women's Studies, 12(1), 128-148.

Kitzie, V., Wagner, T.L., \& Vera, N. (2020). "In the beginning, it was little whispers...Now, we're almost a roar": Conceptualizing a model for community and self in LGBTQ+ health information practices. Paper presented at 2020 iConference, Borås, Sweden, March 23-25.

Knight, R., Karamouzian, M., Carson, A., Edward, J., Carrieri, P., Shoveller, J., . . Fast, D. (2019). Interventions to address substance use and sexual risk among gay, bisexual and other men who have sex with men who use methamphetamine: A systematic review. Drug and Alcohol Dependence, 194, 410-429.

Lerner, J. E., \& Robles, G. (2017). Perceived barriers and facilitators to health care utilization in the United States for transgender people: A review of recent literature. Journal of Health Care for the Poor and Underserved, 28(1), 127-152.

Marcus, B. H., Eaton, C. A., Rossi, J. S., \& Harlow, L. L. (1994). Self-efficacy, decisionmaking, and stages of change: An integrative model of physical exercise. Journal of Applied Social Psychology, 24(6), 489-508. 
RUNNING HEAD: "When it's time to come together, we come together"

McCann, E., \& Brown, M. (2018). The inclusion of LGBT+ health issues within undergraduate healthcare education and professional training programmes: A systematic review. Nurse Education Today, 64, 204-214.

Morgan, A., \& Ziglio, E. (2007). Revitalising the evidence base for public health: An assets model. Promotion \& Education, 14(2_suppl), 17-22.

North Carolina Legislative Assembly House Bill 2. Second Extra Session. 2015-2016 (2016)

Olsson, M. (2005). Beyond 'needy' individuals: Conceptualizing information behavior. Proceedings of the American Society for Information Science and Technology, 42(1).

Pagowsky, N. \& Wallace, N. (2015). Black lives matter!: Shedding library neutrality for social justice. College \& Research Librarians News, 76(4), 196-214.

Reisner, S. L., Poteat, T., Keatley, J., Cabral, M., Mothopeng, T., Dunham, E., . . Baral, S. D. (2016). Global health burden and needs of transgender populations: A review. The Lancet, 388(10042), 412-436.

Romanelli, M., \& Hudson, K. D. (2017). Individual and systemic barriers to health care: Perspectives of lesbian, gay, bisexual, and transgender adults. American Journal of Orthopsychiatry, 87(6), 714-728.

Sampson, R. J., Morenoff, J. D., \& Gannon-Rowley, T. (2002). Assessing “neighborhood effects": Social processes and new directions in research. Annual Review of Sociology, $28(1), 443-478$.

Savolainen, R. (2008). Everyday information practices: A social phenomenological perspective: Lanham, MD: Scarecrow Press. 
RUNNING HEAD: "When it's time to come together, we come together"

Schwarzer, R., \& Renner, B. (2009). Health-specific self-efficacy scales. Freie Universität Berlin. Retrieved from https://userpage.fu-berlin.de/ health/healself.pdf

Schwarzer, R., \& Warner, L. M. (2013). Perceived self-efficacy and its relationship to resilience. In Resilience in children, adolescents, and adults (pp. 139-150). New York, NY: Springer.

Strecher, V. J., McEvoy Devellis, B., Becker, M. H., \& Rosenstock, I. M. (1986). The Role of Self-Efficacy in Achieving Health Behavior Change. Health Education Quarterly, 13(1), 73-92.

Stroumsa, D., Shires, D. A., Richardson, C. R., Jaffee, K. D., \& Woodford, M. R. (2019). Transphobia rather than education predicts provider knowledge of transgender health care. Medical Education, 53(4), 398-407.

Usluel, Y. K. (2007). Can ICT usage make a difference on student teachers' information literacy self-efficacy? Library \& Information Science Research, 29(1), 92-102.

Wagner, T. L., \& Crowley, A. (2020). Why are bathrooms inclusive if the stacks exclude? Systemic exclusion of trans and gender nonconforming persons in post-Trump academic librarianship. Reference Services Review, 48 (1), 159-181.

Wagner, T. L., \& Keeling, S. (2019). Building community-oriented knowledges. In D. Freeburg \& R. D. Lankes (Eds.), Journal of New Librarianship [Knowledge School Edition], 4, $350-360$.

Wagnild, G. (2009). A review of the Resilience Scale. Journal of Nursing Measurement, 17(2), 105-113.

Wagnild, G. M., \& Young, H. (1993). Development and psychometric. Journal of Nursing Measurement, 1(2), 165-178. 
RUNNING HEAD: "When it's time to come together, we come together"

Weinhardt, L. S., Forsyth, A. D., Carey, M. P., Jaworski, B. C., \& Durant, L. E. (1998).

Reliability and validity of self-report measures of HIV-related sexual behavior: Progress since 1990 and recommendations for research and practice. Archives of Sexual Behavior, $27(2), 155-180$

Williams Institute. (2018). LGBTQ people in South Carolina. Retrieved from https://williamsinstitute.law.ucla.edu/wp-content/uploads/South-Carolina-fact-sheet.pdf

Ziglio, E., Hagard, S., \& Griffiths, J. (2000). Health promotion development in Europe: achievements and challenges. Health Promotion International, 15(2), 143-154. 
RUNNING HEAD: "When it's time to come together, we come together"

\section{Appendix A: Supplemental Materials}

\section{Health Information Practices of LGBTQIA+ Communities: http://bit.ly/hiplgbtq}

This weblink to our research page provides a variety of supplemental information to enhance researchers understanding of our chapters findings relating to the health information practices of LGBTQIA+ communities.

- Please visit our site to access study materials such as community presentations, and community forum schedule for LGBTQIA+ communities, and more.

\section{IMLS HIPLGBTQ Interview Protocol: https://bit.ly/2XEzfag}

The above weblink provides open access to our interview protocol used in our study. Access to the document is available for download via Dropbox. 